\title{
Leading Baryon Production at HERA
}

\author{
Vitaliy DODONOV* \\ DESY \\ E-mail: vdodo@mail.desy.de
}

Data from recent measurements of leading baryon production by the $\mathrm{H} 1$ and the ZEUS collaborations are presented and compared to models. The semi-inclusive reactions $e^{+} p \rightarrow e^{+} X p, e^{+} p \rightarrow$ $e^{+} X n$ and $e^{+} p \rightarrow e^{+} j j X n$ are studied using the $\mathrm{H} 1$ and the ZEUS detectors at HERA. Results of three measurements are presented: leading proton production in deep inelastic scattering (DIS) in the kinematic range $Q^{2}>3 \mathrm{GeV}^{2}, 45<W<225 \mathrm{GeV}, p_{T}^{2}<0.5 \mathrm{GeV}^{2}, x_{L}>0.32$; leading neutron production in DIS in the kinematic range $6<Q^{2}<100 \mathrm{GeV}^{2}, 1.5 \cdot 10^{-4}<x<3 \cdot 10^{-2}$, $p_{T}^{2}<0.04 \mathrm{GeV}^{2}, x_{L}>0.32$; dijet production for events with a leading neutron in photoproduction (PHP) in the kinematic range $Q^{2}<1 \mathrm{GeV}^{2}, x_{L}>0.2, p_{T}^{2}<0.476 \cdot x_{L}^{2} \mathrm{GeV}^{2}, E_{T}^{\text {jet } 1}>7.5 \mathrm{GeV}$, $E_{T}^{j e t 2}>6.5 \mathrm{GeV},-1.5<\eta^{\text {jet } 1,2}<2.5$. Standard string fragmentation models do not describe the data. The leading proton production in DIS data are well described by a model including the exchange of both isovector and isoscalar particles, the latter including the pomeron exchange for diffraction. For leading neutron production in DIS and in dijet photoproduction the dominating mechanism for $x_{L} \gtrsim 0.6$ is the pion exchange.

European Physical Society Europhysics Conference on High Energy Physics

July 16-22, 2009

Krakow, Poland

${ }^{*}$ Speaker. 


\section{Introduction}

Events with a baryon carrying a large fraction of the proton beam energy have been observed in $e^{+} p$ scattering at HERA [1, 2]. The mechanisms for leading baryon (LB) production are not completely understood. Leading baryons may be the result of hadronisation of the proton remnant. Exchange of virtual particles is also expected to contribute. In such models, the beam proton fluctuates into a virtual meson-baryon state. The meson scatters with the projectile lepton, leaving the $\mathrm{LB}$ in the final state. Leading neutron $(\mathrm{LN})$ production occurs through the exchange of isovector particles, notably the $\pi^{+}$meson. For leading proton (LP) production isoscalar exchanges also contribute, including diffraction mediated by pomeron exchange.

The H1 and ZEUS experiments at HERA measured leading baryons with dedicated detectors. Leading protons were measured with position sensitive detectors placed along the proton beam downstream of the interaction point. Leading neutrons were measured with lead-scintillator forward neutron calorimeters at the zero-degree point after the proton beam was bent vertically.

The kinematic variables needed to describe the semi-inclusive baryon production are: the virtuality of the exchanged photon $Q^{2}$, the inelasticity $y$, Bjorken- $x$, the $\gamma p$ centre-of-mass energy $W$, the momentum fraction carried by the LB $x_{L}$, and the LB's transverse momentum $p_{T}$.

\section{Leading proton production in DIS}

The ZEUS collaboration presented the measurement of leading proton production in DIS [3]. Events containing LPs with small transverse momentum carrying at least a fraction $x_{L}=0.32$ of the incoming proton momentum were measured in the ZEUS leading proton spectrometer. They were selected from a sample of DIS events with $Q^{2}>3 \mathrm{GeV}^{2}$ and $45<W<225 \mathrm{GeV}$. The LP transverse momentum range was limited to values of $p_{T}^{2}<0.5 \mathrm{GeV}^{2}$. The corresponding integrated luminosity is $12.8 \mathrm{pb}^{-1}$.
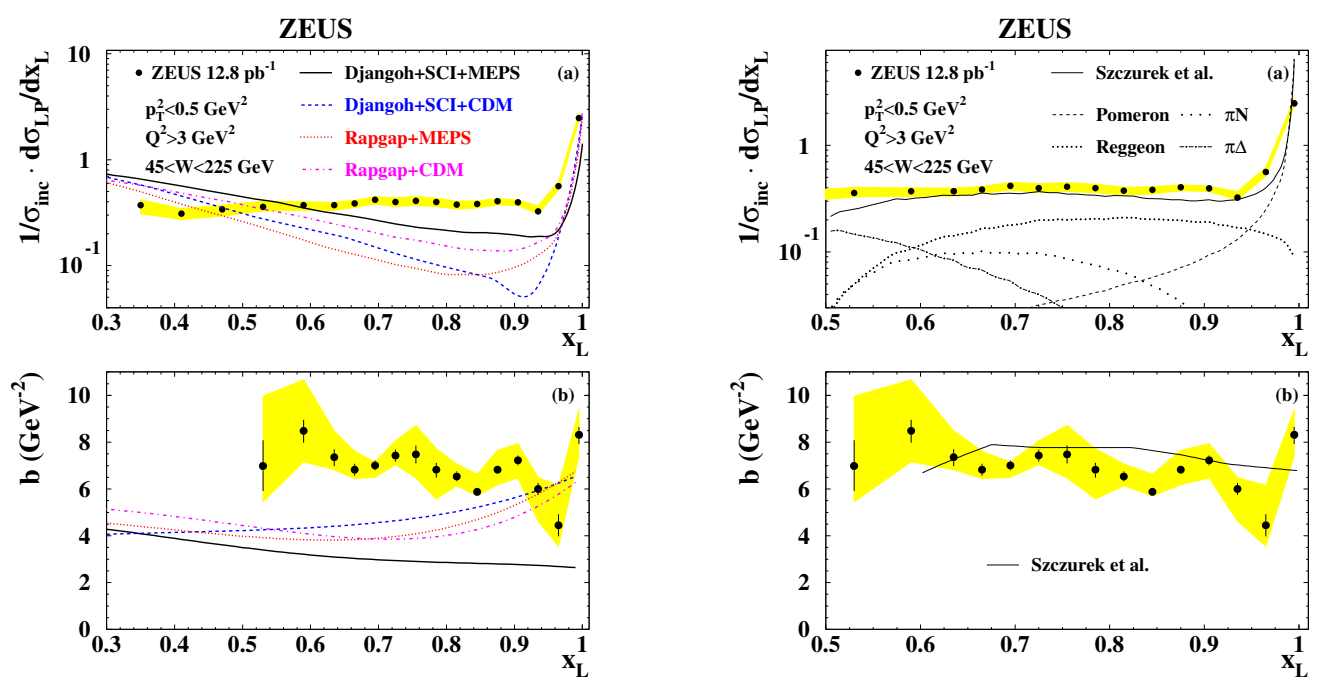

Figure 1: LP $x_{L}$ distribution and exponential slopes compared to standard fragmentation models (left) and a model incorporating isoscalar and isovector exchanges (right). 
Distributions in $p_{T}$ are often parameterised by exponentials $\exp \left(-b p_{T}^{2}\right)$ with a characteristic slope $b$. The left side of Fig. 1 shows a comparison of LP $x_{L}$ distributions and $p_{T}^{2}$ slopes $b$ to the DJANGOH and RAPGAP Monte Carlo models incorporating standard fragmentation and soft colour interactions (SCI), none of which describe the data. The right plots show a comparison to a model including the exchange of both isovector and isoscalar particles, the latter including the pomeron for diffraction [ [ ]. These exchanges together combine to give a good description of the $x_{L}$ distribution and slopes.

\section{Leading neutron production in DIS}

The measurements are performed using $e^{+} p$ data collected in the years 2006 and 2007 with the $\mathrm{H} 1$ detector [5]. The corresponding integrated luminosity is $122 \mathrm{pb}^{-1}$. The kinematic range is $6<Q^{2}<100 \mathrm{GeV}^{2}, 1.5 \cdot 10^{-4}<x<3 \cdot 10^{-2}, 0.32<x_{L}<0.95$ and $p_{T}<0.2 \mathrm{GeV}$.

The programs RAPGAP with $\pi^{+}$-exchange option and DJANGO are used to simulate DIS events. Detector Monte Carlo simulations are used to correct the data for the effects of detector acceptance and QED radiation. The best description of the data is achieved if the Monte Carlo simulations are combined, using weighting factors.

The left side of Fig. 2 shows the measurement of the semi-inclusive structure function $F_{2}^{L N}\left(Q^{2}, x, x_{L}\right)$ for one of the $\left(Q^{2}, x\right)$-bins and it's comparison with the Monte Carlo simulations. For large values of $x_{L}(\gtrsim 0.7)$, the RAPGAP- $\pi$ simulation alone describes the data well. The whole $x_{L}$ range is well described by the combination of RAPGAP- $\pi$ and DJANGO.

Assuming that pion exchange dominates LN production at high $x_{L}$, the pion structure function can be estimated as the ratio $F_{2}^{L N(3)} / \Gamma_{\pi}$, where $\Gamma_{\pi}$ is the integrated over $t$ pion flux. The value of
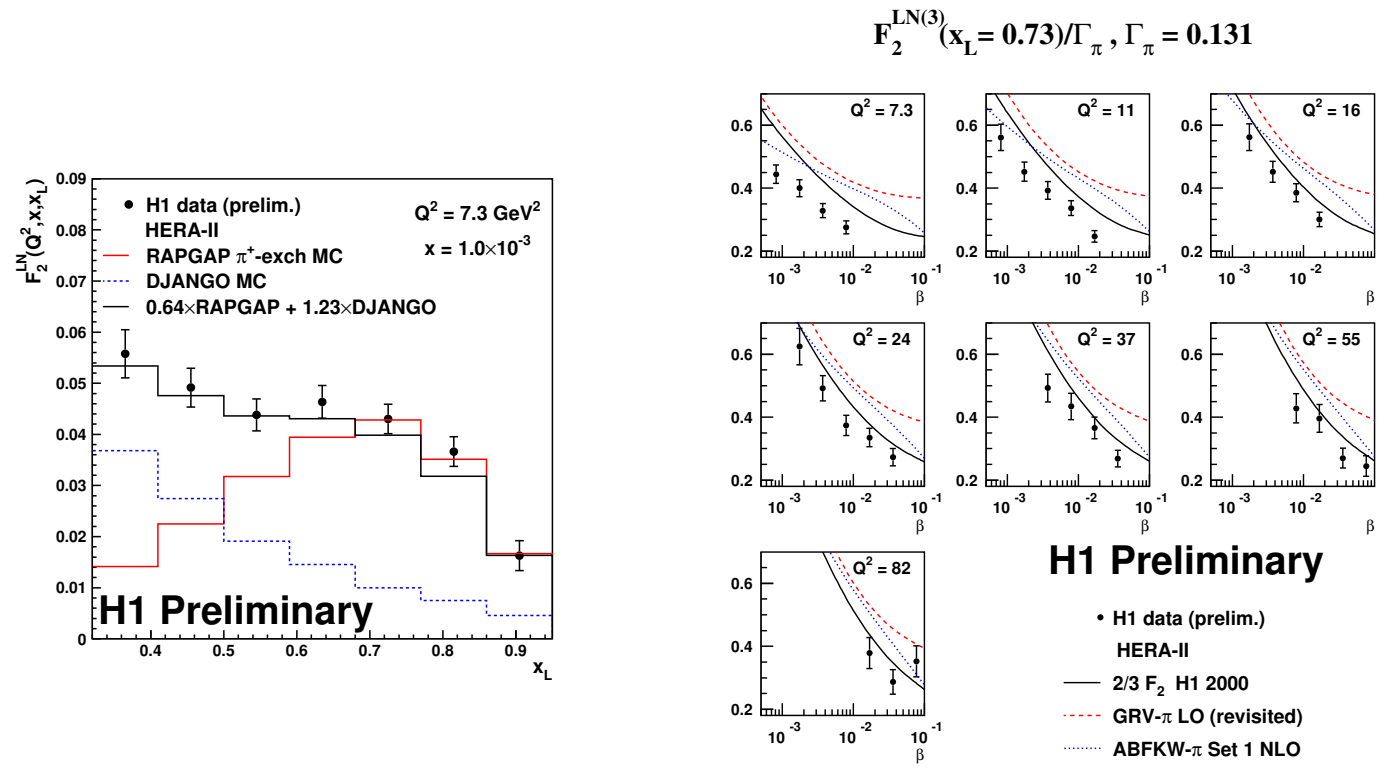

Figure 2: Left: Semi-inclusive LN structure function $F_{2}^{L N(3)}$ in one $\left(Q^{2}, x\right)$-bin compared to the predictions of RAPGAP pion exchange (dashed line) and DJANGO (dotted line) MC simulations. Also shown is a weighted combination of those two simulations (full line). Right: LN structure function $F_{2}^{L N(3)}$ divided by the pion flux $\Gamma_{\pi}$ integrated over $t$ at the average value $x_{L}=0.73$. 
$\Gamma_{\pi}$ depends on pion flux parameterisation. For the pion flux parameterisation from [ø], used here, $\Gamma_{\pi}=0.131$.

The right side of Fig. 2 shows $F_{2}^{L N(3)} / \Gamma_{\pi}$ as a function of $\beta$ for fixed values of $Q^{2}$. The data are compared to the GRV- $\pi$ [7] and ABFKW- $\pi$ [8] parameterisations of the pion structure function, and the HI PDF 2000 fit of the proton structure function [9] scaled by a factor of $2 / 3$.

\section{Leading neutron with dijet in photoproduction}

Differential cross sections for dijet photoproduction (PHP) in association with a leading neutron in the reaction $e^{+}+p \rightarrow e^{+}+n+$ jet + jet $+X$ have been measured with the ZEUS detector using data collected in 1997 [10]. The corresponding integrated luminosity is $40 \mathrm{pb}^{-1}$. The cross sections were measured for jet transverse energies $E_{T}^{\mathrm{jet} 1}>7.5 \mathrm{GeV}, E_{T}^{\mathrm{jet} 2}>6.5 \mathrm{GeV}$, pseudorapidity $-1.5<\eta^{\text {jet1,2 }}<2.5$, photon-proton centre-of-mass energy $130<W<280 \mathrm{GeV}$, momentum fraction carried by the leading neutron $x_{L}>0.2$, and neutron transverse momentum $p_{T}^{2}<0.476 \cdot x_{L}^{2} \mathrm{GeV}^{2}$.
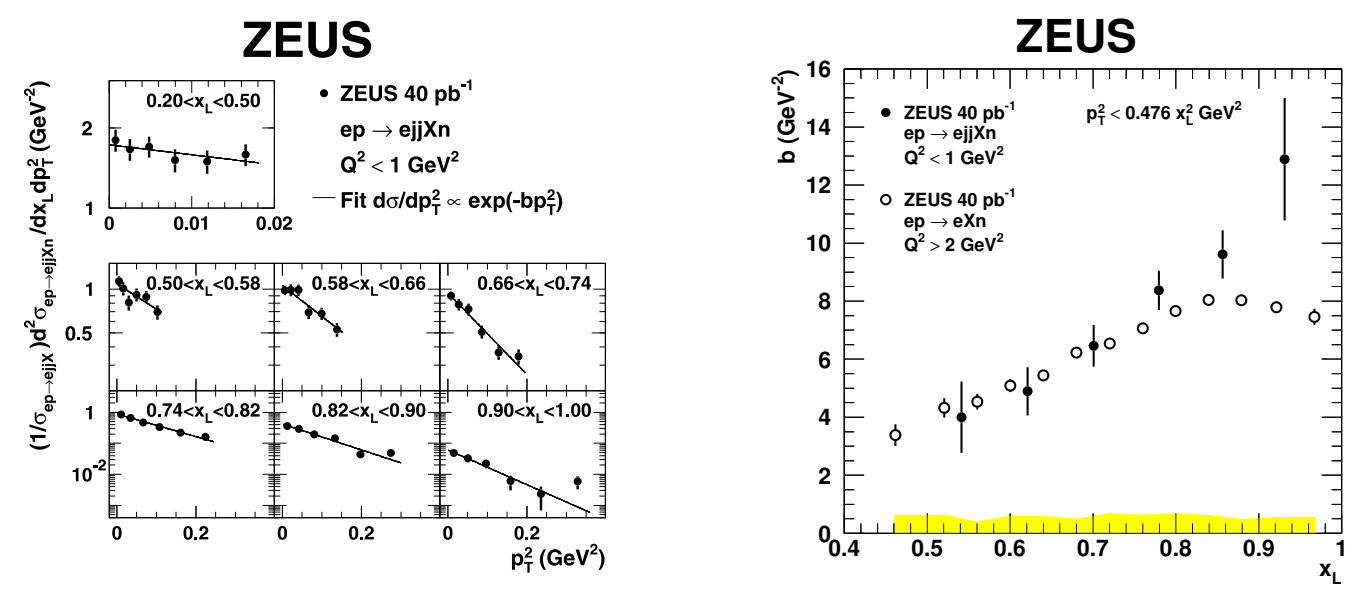

Figure 3: Left: The $p_{T}^{2}$ of leading neutron distributions in bins of $x_{L}$. The line is the result of fit to the form $d \sigma / d p_{T}^{2} \propto \exp \left(-b p_{T}^{2}\right)$. Right: Exponential slopes $b$ from fits of the $p_{T}^{2}$ distributions. The solid points are for dijet photoproduction, the open points are for DIS.

The left side of Fig. 3 shows the $p_{T}^{2}$ distributions for different $x_{L}$ bins. The distributions are compatible with a single exponential. The slopes $b$ of the $p_{T}^{2}$ distributions for dijet PHP and DIS are compared in the right side of Fig. B. Within the limited statistics of the dijet PHP data there is no significant difference between the two sets, suggesting that the neutron production mechanisms for inclusive DIS and dijet PHP are the same as both have hard scale.

The left side of Fig. $\#$ shows the normalised cross sections $\left(1 / \sigma_{e p \rightarrow e j j X}\right) d \sigma_{e p \rightarrow e j j X n} / d x_{L}$. The solid histogram shows the prediction of the full RAPGAP model, the dotted histogram is the contribution from pion exchange, the dashed histogram is the prediction of PYTHIA with the soft colour interaction. RAPGAP- $\pi$-exchange or PYTHIA-SCI alone do not describe data in the full $x_{L}$ range. Full RAPGAP, including standard fragmentation and $\pi^{+}$exchange, describes the data well.

The right side of Fig. 4 shows the comparison of the normalised differential cross sections $\left(1 / \sigma_{i n c}\right) d \sigma_{L N} / d x_{L}$ for dijet PHP, inclusive DIS, and inclusive PHP. Comparing LN $x_{L}$ distribution in PHP and in DIS, the absorption effect is visible in PHP at lower $x_{L}$, as expected in the soft $Q^{2}$ 


\section{ZEUS}

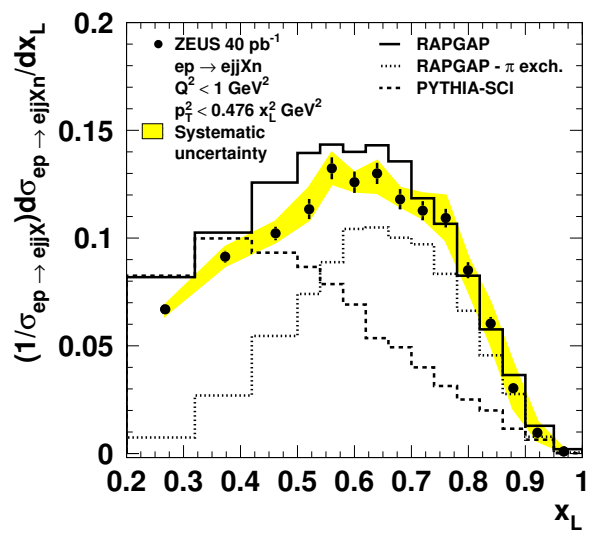

ZEUS

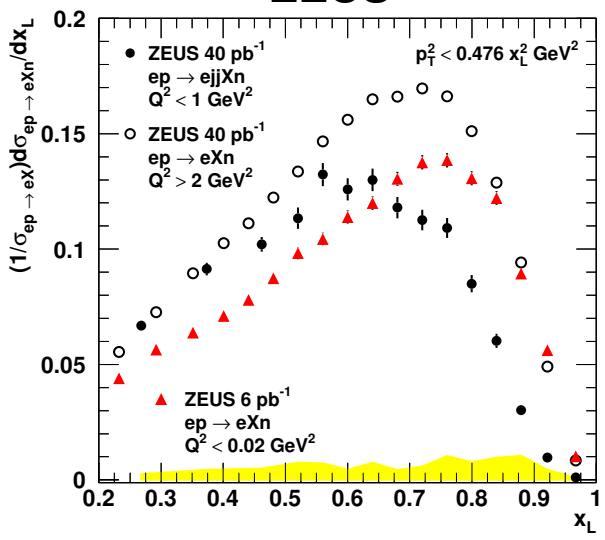

Figure 4: Left: The normalised differential distribution $\left(1 / \sigma_{e p \rightarrow e j j X}\right) d \sigma_{e p \rightarrow e j j X n} / d x_{L}$. The solid histogram shows the prediction of the full RAPGAP model, the dotted histogram is the contribution from pion exchange, the dashed histogram is the prediction of PYTHIA with the SCI. Right: Neutron yields as a function of $x_{L}$ for dijet PHP (solid points), inclusive DIS (open points), and inclusive PHP (shaded triangles).

scale. But for dijet PHP data the absorption is not so prominent. The decrease of the cross section of dijet PHP at higher $x_{L}$ is due to the limited phase space available for LN production in dijet events.

\section{Summary}

The production of leading baryons has been studied as a function of several kinematic variables. There is clear evidence that both contributions from fragmentation processes and from the exchange of colour-neutral particles such as isoscalars are required to describe the data. Thus the data show sensitivity to fragmentation models. The leading neutron data is sensitive to the pion structure function.

\section{References}

[1] A. Aktas et al. [H1 Collaboration], Eur. Phys. J. C 41 (2005) 273 [hep-ex/ 0501074 ].

[2] S. Chekanov et al. [ZEUS Collaboration], Nucl. Phys. B 776 (2007) 1 [hep-ex/ 0702028 ].

[3] S. Chekanov et al. [ZEUS Collaboration], JHEP 0906, (2009) 74 [arXiv: 0812 . 2416 ].

[4] A. Szczurek, N.N. Nikolaev and J. Speth, Phys. Lett. B 428 (1998) 383 [hep-ph / 9712261 ].

[5] H1 Collaboration, Leading Neutron production in DIS at HERA, H1prelim-08-111, http://www-h1.desy.de/h1/www/publications/htmlsplit/H1prelim-08-111.long.html.

[6] H. Holtmann et al., Phys. Lett. B 338 (1994) 363.

[7] M. Glück, E. Reya and I. Schienbein, Eur. Phys. J. C 10 (1999) 313 [hep-ph/9903288].

[8] P. Aurenche et al., Phys. Lett. B 233 (1989) 517.

[9] C. Adloff et al. [H1 Collaboration], Eur. Phys. J. C 21 (2001) 33 [hep-ex/0 012053 ].

[10] S. Chekanov et al. [ZEUS Collaboration], Measurement of dijet photoproduction for events with a leading neutron at HERA, DESY-09-139, to be published in JHEP. 